\title{
A Partial Regularity Result of Harmonic Maps from Manifolds With Bounded Measurable Riemannian Metrics
}

\author{
SHI YUGUANG
}

\section{Introduction.}

In the last two decades,much progress has been made on the regularity theory of harmonic maps between manifolds,for instance,one can see [GG1], [GG2], [SU],[E],[B] etc. Since the methods used in these papers are variations of Schauder's device of freezing the coefficients they need assume that the metrics are at least uniformly continuous while in [SU],[E],[B] they assume $g \in C^{2}(M)$. If the image of a map is contained in a convex ball; the metric of the domain is only bounded measurable,Hildebrandt-Widman proved the map is Hölder continuous(see[H-W]). On the other hand,M.Gromov and $R$. Schoen established the existence and regularity theory of harmonic maps into singular space(see [GS]). It may be more interesting, in view of analysis, to consider the same problem when the domain is singular. In this paper, we shall consider this problem and prove partial regularity results of energy minimizing maps from manifolds with bounded measurable Riemannian metrics. We will consider the more complicated case,for example, the domain is a algebraic variety, in elsewhere.

Suppose $M$ is an $n$-dimensional differentiable manifold, $g$ is a metric on $M$, then $g$ can be represented by a positive definite matrix $\left(g_{i j}\right)$ in each coordinate chart $\left(\mathcal{D}, x^{i}\right)$ of $M$.If $g_{i j}$ merely belongs to $L^{\infty}(\mathcal{D}, \mathbf{R})$, then $g$ is called bounded measurable Riemannian metric,or simply $L^{\infty}$-metric.Let $(N, h)$ be a smooth compact Riemannian manifold, by the Nash embedding theorem, we can always assume $(N, h)$ is isometrically embedded in some Euclidean space $\mathbf{R}^{k}$.

In order to discuss harmonic maps from $(M, g)$ to $(N, h)$, we work in the seperable Hilbert space $L_{1}^{2}\left(M, \mathbf{R}^{k}\right)$, the set of maps $u: M \longmapsto \mathbf{R}^{k}$, whose component functions have first derivatives in $L^{2}$. As in the smooth case, 
we can define the space $L_{1,0}^{2}\left(M, \mathbf{R}^{k}\right)$;energy functional $E($.$) on the space$ $L_{1}^{2}\left(M, \mathbf{R}^{k}\right) ; E$-minimizing map from $(M, g)$ to $(N, h)$, (see [SU],pp309).

Since we are interested in the regularity theory, we assume $(M, g)$ is simply $\left(B_{1}, g\right)$ where $B_{1}$ is the unit ball in $\mathbf{R}^{n}, g$ is an $L^{\infty}$ - metric on it. For convenience,we identify the matrix $\left(g_{i j}\right)$ representing $g$ in standard coordinates $\left(x^{1}, \ldots, x^{n}\right)$ of $\mathbf{R}^{n}$ with $g$ itself. In addition we adopt the notation:

$$
|\nabla u|^{2}=\sum_{1 \leq \alpha \leq n} \sum_{1 \leq i \leq k}\left(\frac{\partial u^{i}}{\partial x^{\alpha}}\right)^{2} .
$$

The main result of this paper is the following:

Theorem 1. Suppose $B_{r}$ is a ball in $\mathbf{R}^{n}$ with radius $r, g$ is an $L^{\infty}{ }_{-m e t r i c}$ on $B_{1}$, with $\Lambda^{-1} I \leq g \leq \Lambda I$, where $\Lambda$ is a positive constant, $I$ is the unit matrix. Assume $(N, h)$ is a smooth compact Riemannian manifold, $u$ is an $E$-minimizing map from $\left(B_{1}, g\right)$ to $(N, h)$. Then there exists a $\varepsilon=\varepsilon(\Lambda)>$ 0 , such that if $\int_{B_{1}}|\nabla u|^{2} d x \leq \varepsilon^{2}$, then $u \in C^{\alpha}\left(B_{1 / 2}, \mathbf{R}^{k}\right)$ for some $0<\alpha<1$.

Remark 0.1. Meyers'example (see [G],pp 157-158) shows that the $c^{\alpha}-$ regularity in the theorem is optimal.

As a corollary of Theoem 1, we have:

Theorem 2. Let Sing $(u)$ be the set $\{x \in M: u$ is discontinous at $x\}$, and $\mathcal{H}^{n-2}$ be $(n-2)$-dimensional Hausdorff measure, then $\mathcal{H}^{n-2}($ Sing $u)=0$.

We follow the approach of Hardt and Lin in [HL].However,there is a major difficulty in this approah when the metric is merely measurable,i.e. one cannot do the domain blow-ups and take the limit.To overcome this difficulty, we use the Green functions(cf.[H-K-W],[H-W]) to get the necessary estimate.

The rest of the paper runs as follows: In $\S 1$, we will introduce some notations and prove Lemmas.In $\S 2$ we will prove energy improvement theorem (Theorem 2.1).Once the energy improvement is obtained, one can prove Theorem 1 and 2 easily by standard technique,for the details see [SU] or $[\mathrm{HL}]$,we will omit them in this paper.

\section{Preliminaries.}

Let $a^{\alpha \beta}=g^{\alpha \beta}\left(\operatorname{det}\left(g_{\alpha \beta}\right)\right)^{1 / 2}$, where $\left(g^{\alpha \beta}\right)$ denotes the inverse matrix of $\left(g_{i j}\right)$. Without loss of generality, we may assume $\Lambda^{-1} I \leq\left(a^{\alpha \beta}\right) \leq \Lambda I$. 
We say that $u$ is a minimizer under $a^{\alpha \beta}$ if $\mathrm{u}$ is an $E$-minimizing map from $\left(B_{1}, g\right)$ to $(N, h)$.It is easy to verify that, u satisfies the equations:

$$
-\frac{\partial\left(a^{\alpha \beta} \frac{\partial u}{\partial x^{\beta}}\right)}{\partial x^{\alpha}}=A(u)(\nabla u, \nabla u) \quad \text { in } B_{1} .
$$

where $A$ is the second fundamental form of $N$ in $\mathbf{R}^{k}$.

Let $\mathcal{F}_{\Lambda}=\left\{\left(a^{\alpha \beta}\right): \Lambda^{-1} I \leq\left(a^{\alpha \beta}\right) \leq \Lambda I\right.$ in $\left.B_{1}\right\}$. Clearly, $\mathcal{F}_{\Lambda}$ is invariant under rescaling and translating. i.e. $\left(a^{\alpha \beta}(\lambda x+y)\right) \in \mathcal{F}_{\Lambda}$ whenever $\left(a^{\alpha \beta}\right) \in$ $\mathcal{F}_{\Lambda}$ and $\lambda x+y \in B_{1}$.

Lemma 1 ([HL],Corollary 2.3). There exist postive constants $c$ and $q$ such that if $0<\lambda<1 / 4$, and $u$ is an E-minimizer in $L_{1}^{2}(B, N)$ with $E_{1}(u)=$ $\int_{B_{1}}|\nabla u|^{2} d x \leq c^{-1} \lambda^{q / 2}$, then

$$
E_{1 / 2}(u) \equiv(1 / 2)^{2-n} \int_{B_{1 / 2}}|\nabla u|^{2} d x \leq \lambda E_{1}(u)+c \lambda^{-q} f_{B_{1}}|u-\bar{u}|^{2} d x
$$

where $\bar{u}=f_{B} u d x=\frac{1}{|B|} \int_{B} u d x$.

Main Lemma. Let $\left(a_{k}{ }^{\alpha \beta}\right) \in \mathcal{F}_{\Lambda}, k=1,2, \ldots$. Assume that $v_{k} \in L_{1}^{2} \cap$ $L^{\infty}\left(B_{1}, \mathbf{R}^{k}\right)$ and $\int_{B_{1}}\left|\nabla v_{k}\right|^{2} d x \leq 1$. Suppose that

$$
-\frac{\partial\left(a_{k}^{\alpha \beta}\left(\frac{\partial v_{k}}{\partial x^{\beta}}\right)\right)}{\partial x^{\alpha}}=h_{k}
$$

and $w_{k}$ is the solution of:

$$
\left\{\begin{array}{l}
-\frac{\partial\left(a_{k}^{\alpha \beta} \frac{\partial w_{k}}{\partial x^{\beta}}\right)}{\partial x^{\alpha}}=0 \\
\left.w_{k}\right|_{\partial B_{1}}=\left.v_{k}\right|_{\partial B_{1}}
\end{array}\right.
$$

If $h_{k}$ converges strongly to 0 in $L^{1}\left(B_{1}, \mathbf{R}^{k}\right)$ as $k \rightarrow \infty$, then $f_{k} \equiv w_{k}-v_{k}$ converges weakly to 0 in $L_{1}^{2}\left(B_{1}, \mathbf{R}^{k}\right)$.

Proof. Let $G_{k}(x, y)$ be the Green function of operator:

$$
L_{k}(\cdot) \equiv-\frac{\partial\left(a_{k}{ }^{\alpha \beta} \frac{\partial(\cdot)}{\partial x^{\beta}}\right)}{\partial x_{\alpha}}
$$


and $G_{k}^{\sigma}(x, y)=f_{B_{\sigma}(y)} G_{k}(x, z) d z$, then $G_{k}(x, y)$ and $G_{k}^{\sigma}(x, y)$ enjoy the following properties, see [GW].

i) $0 \leq G_{k}(x, y) \leq c|x-y|^{2-n}, \quad c=c(n, \Lambda)$.

ii) $\int_{B_{1}} a_{k}{ }^{\alpha \beta} \frac{\partial \varphi}{\partial x^{\alpha}} \frac{\partial G_{k}^{\sigma}(x, y)}{\partial x^{\beta}} d x=\int_{B_{\sigma(y)}} \varphi d x, \forall \varphi \in L_{1,0}^{2} \cap L^{\infty}\left(B_{1}, \mathbf{R}^{k}\right)$, $\forall B_{\sigma}(y) \subset B_{1}$.

iii) $G_{k}^{\sigma}(x, y) \in L_{1,0}^{2} \cap L^{\infty}\left(B_{1}, \mathbf{R}\right),\left\|G_{k}^{\sigma}(x, y)\right\|_{L^{\infty}\left(B_{1}, \mathbf{R}^{k}\right)} \leq c(\sigma)$,for any fixed $y \in B_{1}$.

By $\left({ }^{*}\right)\left({ }^{* *}\right)$ we have

$$
-\frac{\partial\left(a_{k}^{\alpha \beta}\left(\frac{\partial\left(v_{k}-w_{k}\right)}{\partial x^{\beta}}\right)\right)}{\partial x^{\alpha}}=h_{k} .
$$

One can show that $v_{k}-w_{k} \in L_{1,0}^{2} \cap L^{\infty}\left(B_{1}, \mathbf{R}^{k}\right)$ with the help of the maximum principle. Taking $G_{k}^{\sigma}(x, y)$ as a test function of the above equations and noting the propertity (ii) of $G_{k}^{\sigma}(x, y)$ we get:

$$
f_{B_{\sigma}(y)}\left(v_{k}-w_{k}\right) d x=\int_{B_{1}} h_{k} \cdot G_{k}^{\sigma}(x, y) d x \quad \forall y \in B_{1-2 \sigma}
$$

hence,

$$
\int_{B_{1-2 \sigma}}\left|f_{B_{\sigma}(y)}\left(v_{k}-w_{k}\right) d x\right| d y \leq \int_{B_{1-2 \sigma}} \int_{B_{1}}\left|h_{k}\right| \cdot G_{k}^{\sigma}(x, y) d x d y .
$$

Due to property (iii) of $G_{k}^{\sigma}(x, y)$, we can obtain:

$$
\int_{B_{1-2 \sigma}}\left|f_{B_{\sigma}(y)} f_{k} d x\right| d y \leq c(\sigma) \int_{B_{1}}\left|h_{k}\right| d x \rightarrow 0, \text { as } \quad k \rightarrow \infty .
$$

However, it is easy to see, for every sequence $\left\{f_{k_{i}}\right\} \subset\left\{f_{k}\right\}$, there exists a subsequence of $\left\{f_{k_{i}}\right\}$ which is also denoted by $\left\{f_{k}\right\}$, and a $f_{0} \in L_{1,0}^{2}\left(B_{1}, \mathbf{R}^{k}\right)$ such that $f_{k}$ converges weakly to $f_{0}$ in $L_{1,0}^{2}\left(B_{1}, \mathbf{R}^{k}\right)$ and $f_{k}$ converges strongly to $f_{0}$ in $L^{2}\left(B_{1}, \mathbf{R}^{k}\right)$. Thus, we arrive at:

$$
\int_{B_{1-2 \sigma}}\left|f_{B_{\sigma}(y)} f_{0} d x\right| d y=0, \quad \text { for every } y \in B_{1-2 \sigma} .
$$

Since $\sigma$ is arbitrary in $(0, \delta)$, for some small positive $\delta$, the above equality implies $f_{0} \equiv 0$ in $B_{1}$, the Main Lemma follows. 


\section{Proof of Energy improvement Theorem.}

Theorem 2.1 (Energy-improvement). There exists $\theta$ and $\epsilon$ with $0<\theta=$ $\theta(\Lambda)<1 / 2, \epsilon=\epsilon(\Lambda)>0$, such that if $u$ is a minimizer under $a^{\alpha \beta}$, where $\left(a^{\alpha \beta}\right) \in \mathcal{F}_{\Lambda}$.then we have

$$
\theta^{2-n} \int_{B_{\theta}}|\nabla u|^{2} d x \leq 1 / 2 \int_{B_{1}}|\nabla u|^{2} d x .
$$

Provided $\int_{B_{1}}|\nabla u|^{2} d x \leq \epsilon^{2}$.

Proof. Were the thoerem false, there would exist, for each $\theta$ with $0<\theta<$ $1 / 2$,a sequence $\left\{u_{k}\right\}$ of minimizers under $\left(a_{k}^{\alpha \beta}\right) \in \mathcal{F}_{\Lambda}$, such that:

$$
\int_{B_{1}}\left|\nabla u_{k}\right|^{2} d x=\epsilon_{k}^{2} \rightarrow 0 \text { as } k \rightarrow \infty, \text { and } \theta^{2-n} \int_{B_{\theta}}\left|\nabla u_{k}\right|^{2} d x \geq 1 / 2 \epsilon_{k}^{2}
$$

we may form an associated normlized sequence:

$$
v_{k}=\frac{u_{k}-c_{k}}{\epsilon_{k}}
$$

where $c_{k}$ is a constant vector which will be determined later.

Clearly,

$$
\begin{gathered}
\int_{B_{1}}\left|\nabla v_{k}\right|^{2} d x=1 \\
\theta^{2-n} \int_{B_{\theta}}\left|\nabla v_{k}\right|^{2} d x \geq 1 / 2
\end{gathered}
$$

and

$$
-\frac{\partial\left(a_{k}^{\alpha \beta}\left(\frac{\partial v_{k}}{\partial x^{\beta}}\right)\right)}{\partial x^{\alpha}}=\epsilon_{k} A\left(u_{k}\right)\left(\nabla v_{k}, \nabla v_{k}\right) \quad \text { for every } k \geq 1 .
$$

We will prove this theorem by showing $\theta^{2-n} \int_{B_{\theta}}\left|\nabla u_{k}\right|^{2} d x \leq 1 / 4 \epsilon_{k}^{2}$ when $\theta$ is sufficently small, and $k$ is sufficiently large.

First,as in [HL], we can apply Lemma 1 to get the estimate (see [HL] pp564):

$$
\begin{aligned}
\theta^{2-n} \int_{B_{\theta}}\left|\nabla u_{k}\right|^{2} d x \leq & \lambda^{j}\left(2^{j} \theta\right)^{(2-n)} \int_{B_{2^{j}}}\left|\nabla u_{k}\right|^{2} d x \\
& +c \lambda^{-q} \sum_{h=0}^{j-1} \lambda^{h} \int_{B_{2^{h+1} \theta}}\left|u_{k}-\bar{u}_{k}^{(h+1)}\right|^{2} d x
\end{aligned}
$$


for sufficiently large $k$ (may depend on $\theta$ ), where $\bar{u}_{k}^{h}=f_{B_{2^{h} \theta}} u_{k} d x$, and $j$ is a positive integer such that $1 / 4 \leq 2^{j} \theta \leq 1 / 2$.

Secondly, we want to show:

$$
f_{B_{2^{h} \theta}}\left|u^{k}-\bar{u}_{k}{ }^{(h)}\right|^{2} d x \leq c\left(2^{h} \theta\right)^{2 \alpha} \varepsilon_{k}{ }^{2}, \text { provided } \mathrm{k} \text { is large enough. }
$$

where $0 \leq h \leq j, 0<\alpha<1$, and $c$ is constant independents of $k$.

Suppose $r \in(\theta, 1 / 2)$, take $c_{k}=\bar{u}_{k}(r) \equiv f_{B_{r}} u_{k} d x$ in the definition of $v_{k}$, and set:

$$
v_{k}^{r}=\frac{u_{k}-\bar{u}_{k}(r)}{\varepsilon_{k}} .
$$

Let $w_{k}^{r}$ denote the solution of the equations

$$
\left\{\begin{array}{l}
-\frac{\partial\left(a_{k}^{\alpha \beta}\left(\frac{\partial w_{k}^{r}}{\partial x^{\beta}}\right)\right)}{\partial x^{\alpha}}=0 \\
w_{k}^{r}-\left.v_{k}^{r}\right|_{\partial B_{1}}=0
\end{array} \quad \text { in } \quad B_{1} .\right.
$$

By the definition of $v_{k}^{r}$ and Main Lemma,it is easy to see $f_{k}^{r} \equiv w_{k}^{r}-v_{k}^{r}$ converges weakly to 0 in $L_{1,0}^{2}\left(B_{1}, \mathbf{R}^{k}\right), f_{k}^{r}$ converges strongly to 0 in $L^{2}\left(B_{1}, \mathbf{R}^{k}\right)$ and $\overline{w_{k}^{r}}=f_{B_{r}} w_{k}^{r} d x \rightarrow 0$ as $k \rightarrow \infty$.

Next,we will show $\int_{B_{r}}\left|v_{k}^{r}\right|^{2} d x \leq c r^{2 \alpha}$ when $k \geq k(\theta)$ is sufficiently large. Obviously we have:

$$
f_{B_{r}}\left|v_{k}^{r}\right|^{2} d x \leq 4\left(f_{B_{r}}\left|v_{k}^{r}-w_{k}^{r}\right|^{2} d x+f_{B_{r}}\left|w_{k}^{r}-\overline{w_{k}^{r}}\right|^{2} d x+\left|\overline{w_{k}^{r}}\right|^{2}\right) .
$$

Notice that $w_{k}^{r}$ is the solution of (2),we can obtain ( see [G],Theorem 21 pp.53):

$$
\left|w_{k}^{r}(x)-w_{k}^{r}(y)\right| \leq c(n, \Lambda)|x-y|^{\alpha},
$$

for every $x, y \in B_{1 / 2}$, here $\alpha=\alpha(\Lambda, n)$, then:

$$
\left|w_{k}^{r}(x)-\overline{w_{k}^{r}}\right| \leq f_{B_{r}}\left|w_{k}^{r}(x)-w_{k}^{r}(y)\right| d y \leq c(n, \Lambda)(2 r)^{\alpha} .
$$

Since $v_{k}^{r}-w_{k}^{r}$ converges strongly to 0 in $L^{2}\left(B_{1}, \mathbf{R}^{k}\right)$ and

$$
\overline{w_{k}^{r}} \rightarrow 0 \text { as } k \rightarrow \infty ; r \geq \theta>0 \text {, we get: }
$$

$$
f_{B_{r}}\left|v_{k}^{r}\right|^{2} d x \leq c r^{2 \alpha}
$$


or

$$
f_{B_{r}}\left|u_{k}^{r}-\bar{u}_{k}(r)\right|^{2} d x \leq c r^{2 \alpha} \varepsilon_{k}^{2},
$$

when $k$ is sufficently large, where $c=c(n, \Lambda)$.

Choosing $r=2^{h} \theta$, where $0 \leq h \leq j$, we can see that, for any fixed $\theta>0$,if $k \geq k(\theta)$ is sufficiently large, there holds:

$$
f_{B_{2^{h} \theta}}\left|u_{k}-{\overline{u_{k}}}^{(h)}\right|^{2} d x \leq c\left(2^{h} \cdot \theta\right)^{2 \alpha} \cdot \varepsilon_{k}^{2} .
$$

Finally,it follows by substituting (3) into (1) and letting $k \rightarrow \infty$; that

$$
\theta^{2-n} \int_{B_{\theta}}\left|\nabla u_{k}\right|^{2} d x \leq c \cdot \varepsilon_{k}^{2}\left(\lambda^{j}+\theta^{2 \alpha} \cdot \lambda^{-q}\right), \text { where } c=c(n, \Lambda) .
$$

Choosing $\theta$ sufficiently small such that $c \cdot\left(\theta^{2 \alpha} \lambda^{-q}+\lambda^{j}\right) \leq 1 / 4$ (since $1 / 4 \leq$ $\left.2^{j} \theta \leq 1 / 2\right)$ we can get the estimate:

$$
\theta^{2-n} \int_{B_{\theta}}\left|\nabla u_{k}\right|^{2} d x \leq 1 / 4 \varepsilon_{k}^{2}
$$

which contradicts the original choice of $u_{i}$. This completes the proof of Theorem 2.1 .

Acknowledgments. The author would like to express his gratitude to his advisor Prof. Ding Weiyue and Prof. Lin Fanghua for their suggesting this problem and advising during the preparation.

\section{References.}

[B] F.Bethuel, On the Singular set of stationary harmonic Maps, Manuscrpta Math. 78 (1993), 417-443.

[E] C.L.Evans, Partial regularity for stationary harmonic maps into sphere, Arch. Rat. Mech. Anal. 116 (1991), 101-163.

[G] M.Giaquinta, Multiple integrals in the calculus of variations and non -linear elliptic systems, Princeton Univ. Press (1983).

[GG1] M.Giaquinta and M.E.Giusti, Nonlinear elliptic systems with quadratic growth, Manuscripta Math. 24 (1978), 323-349. 
[GG2] M.Giaquinta and M.E.Giusti, On the regularity of minima of variational integrals, Acta. Math. 148 (1982), 31-46.

[GS] M.Gromov and R.Schoen, Harmonic Maps into Singular Space and padic superrigidity for lattices in group of Rank one, Publ. IHES 1993.

[GW] M.Gruter and K.O.Widman, The Green function for uniformly Elliptic Equation, Manuscripta. Math. 37 (1982), 303-342.

[H-K-W] S.Hildebrandt, H.Kaul and K.O. Widman, An existence theorem for harmonic mappings of Riemannian manifolds, Acta. Math. 138 (1978), 116.

[H-W] S.Hildebrandt and K.O.Widman, On the Hölder Continuity of weak Solutions of Quasilinear Elliptic Systems Of Second Order, Ann. Sc. Norm. Pisa, IV (1977), 145-178.

[HL] R.Hardt and F.H.Lin, Mapping Minimizing the $L^{p}$-norm of the Gradient, C.P.A.M. Vol. XL (1987), 555-588.

[SU] R.Schoen and K.Uhlenbeck, A regularity theory for Harmonic Maps, J. Differential Geometry, 17 (1982), 307-335.

ReCeived December 8Th, 1994 AND Revised April 28Th, 1995.

INSTITUTE OF MATHEMATICS

ACADEMIA SiniCA

BEIJING 100080 